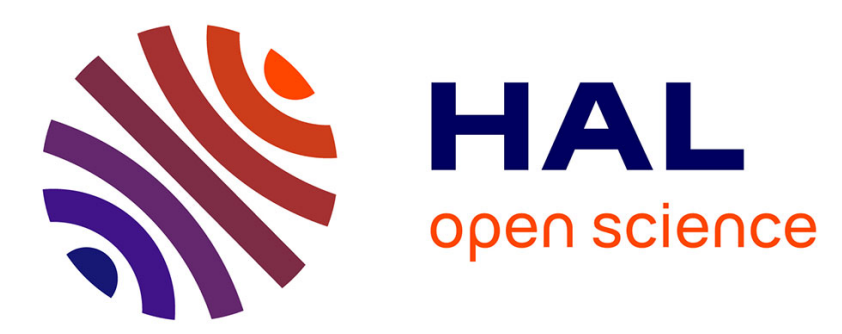

\title{
Intermittent flow of a collection of rigid frictional disks in a vertical pipe
}

Ivar Bratberg, Farhang Radjai, Alex Hansen

\section{To cite this version:}

Ivar Bratberg, Farhang Radjai, Alex Hansen. Intermittent flow of a collection of rigid frictional disks in a vertical pipe. Physical Review E: Statistical, Nonlinear, and Soft Matter Physics, 2005, 71, pp.011301. 10.1103/PhysRevE.71.011301 . hal-00759655

\section{HAL Id: hal-00759655 \\ https://hal.science/hal-00759655}

Submitted on 2 Jun 2021

HAL is a multi-disciplinary open access archive for the deposit and dissemination of scientific research documents, whether they are published or not. The documents may come from teaching and research institutions in France or abroad, or from public or private research centers.
L'archive ouverte pluridisciplinaire HAL, est destinée au dépôt et à la diffusion de documents scientifiques de niveau recherche, publiés ou non, émanant des établissements d'enseignement et de recherche français ou étrangers, des laboratoires publics ou privés. 


\title{
Intermittent flow of a collection of rigid frictional disks in a vertical pipe
}

\author{
I. Bratberg* \\ Department of Telecommunications, Norwegian University of Science and Technology, N-7491 Trondheim, Norway \\ F. Radjai \\ LMGC, CNRS-Université Montpellier II, Place Eugne Bataillon, 34095 Montpellier cedex, France
}

A. Hansen

Department of Physics, Norwegian University of Science and Technology, N-7491 Trondheim, Norway

(Received 30 June 2003; revised manuscript received 11 October 2004; published 5 January 2005)

\begin{abstract}
We study the quasistatic flow of a collection of rigid frictional disks pushed upward (against the gravity) inside a narrow vertical pipe by a compliant mechanism. The contact dynamics method was used for the numerical simulations in combination with a friction law at disk-disk and wall-disk contacts characterized by discontinuous velocity weakening from a static threshold to a dynamic coefficient of friction. The material is sheared by the rolling of particles at the walls inducing a convective motion in the bulk. We observe a transition from constant flow to an intermittent flow when the driving velocity is reduced below a characteristic velocity that scales as $k^{-1 / 2}$ with the stiffness $k$ of the pushing mechanism. The intermittent flow is composed of alternating phases of creep motion, where the pressure at the bottom of the granular column rises nonlinearly with time, and sudden slip, corresponding to a fast pressure drop. We show that the mean static pressure is correctly predicted by the Janssen model. The interplay between friction mobilization at the walls and structural changes in the bulk gives rise to a broad distribution of slip amplitudes characterized by a power law with an exponent $\simeq-1.7$ that appears to be robust with respect to our system parameters.
\end{abstract}

DOI: 10.1103/PhysRevE.71.011301

PACS number(s): $83.80 . \mathrm{Fg}, 45.70 . \mathrm{Mg}$

\section{INTRODUCTION}

The slow continuous loading of a granular material leads often to unstable failure with collective rearrangements that dissipate unevenly part of the potential energy stored in the system. Slope failure is a well-known example of such a transition. Similar behavior has been reported for a granular packing slowly pushed by a solid object [1,2], a granular bed sheared by the motion of a plate on top of it $[3,4]$, a granular column pushed vertically from the bottom in a pipe $[5,6]$, a powder sheared in an annular cell [7], an array of cylinders pushed on a plane [8], and gravitational flow around a solid suspended plate [9]. Different origins have been attributed to this unstable onset of granular motion: inertial effects [10], dilatancy [11], jamming-unjamming transition based on the observation of force chains [2], arching and blocking enhancement due to the presence of the walls [5]. All these mechanisms refer basically to the frustration of particle motions induced by friction and rigidity of the particles that are possibly enhanced by confining walls.

Unstable failure implies that granular friction (characterizing the resistance of a granular material to shear) declines with failure in strong analogy with sliding instability of solid bodies [12-14] where the friction force at the contact between two solid bodies is a decreasing function of sliding velocity at low velocities. The most basic description of ve-

\footnotetext{
*Present address: Fysisk Institutt, Universitetet i Oslo, Postboks 1048 Blindern, N-0316 Oslo, Norway.

Electronic address: ivar.bratberg@fys.uio.no
}

locity weakening friction is provided by simply assuming different static and dynamic coefficients of friction for the material corresponding, respectively, to the resistance before and after failure. This assumption leads to a periodic "stickslip" oscillation in the steady state [15]. In practice, due to granular disorder, some degree of randomness is observed in the sizes of successive loading-unloading events (slip amplitude, pressure drop, sticking time, energy release, avalanche size, etc.).

The distribution of event sizes is generally broad but is often peaked on a rather well-defined value. The search for a power-law distribution of event sizes in granular experiments is motivated either by the observation of power laws in seismic dynamics along faults [16] or as a realization of the popular concept of self-organized criticality [17]. Experimental observation of power laws in avalanche sizes for piles of elongated grains (rice piles) suggests that event size distributions can be made increasingly broad by reducing inertial effects and increasing dissipation in particle interactions $[18,19]$. Very irregular stick-slip motion with a nearly powerlaw distribution of energy release was observed also in the experiments by Kolb et al. where a granular column composed of highly frictional beads was pushed upward inside a two-dimensional cell [5]. The grains, due to their weights, tend to form upward arches that cannot resist upward motion or redistribute efficiently the extra pressure from the pushing mechanism to the walls. As a result, the upward motion tends to destabilize arches whereas wall friction is polarized downward, i.e., against the upward motion. This suggests that the main source of velocity weakening in these experiments is likely to be at the contacts with the walls. 


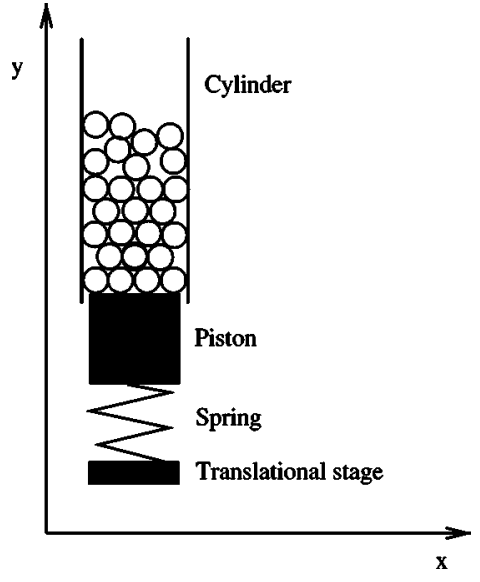

FIG. 1. Representation of the numerical setup.

In this paper, we study a similar setup by means of contact dynamics simulations. The main idea of these simulations was to keep the system in the steady state for a long time in order to obtain reliable statistics for the analysis of slip events. For an efficient shearing of the granular column we used a larger coefficient of friction for wall-particle than for particle-particle contacts. As a result the particles tend mostly to roll on the walls and they induce a convective motion in the bulk. We observe an intermittent flow only when the contacts are governed by a velocity weakening friction law and when the driving velocity is below a characteristic velocity. We compare the mean force with the prediction of a simple Janssen analysis and in connection with friction mobilization at the walls and normal stress ratio inside the material. We analyze the fluctuations of slip events and their correlation with structural changes in the packing. Interestingly, we find a power-law distribution for slip amplitudes over three decades. The value of the exponent is studied as a function of system parameters and shown to be quite robust.

\section{SIMULATED SYSTEM}

The setup is represented in Fig. 1. The cell consists of two fixed vertical (parallel to the $y$ axis) straight lines separated by a distance $L$ and playing the role of walls and a horizontal straight line that can move vertically and plays the role of a piston. The latter is connected via a linear spring of stiffness $k$ to a translational stage moving at a constant upward velocity $v$. The cell is filled with particles up to a height $H$. The particles are disks with a uniform distribution of their diameters between $D_{\min }=0.75 \mathrm{~mm}$ and $D_{\max }=1 \mathrm{~mm}$. The particles, the walls and the piston are assumed to be infinitely rigid so that the pushing spring is the only part of the system that can store elastic energy. We also assume that the contacts interact through a Coulomb friction law with a static coefficient of friction $\mu_{s}$ and a dynamic coefficient of friction $\mu_{d}$ with $\mu_{s}>\mu_{d}$. The graph relating the friction force $T$ at a contact to the sliding velocity $v_{s}$ is shown in Fig. 2. This graph corresponds to a discontinuous velocity weakening friction. As long as $v_{s}=0$, the friction force $T$ can take any value in the range $\left[-\mu_{s} N, \mu_{s} N\right]$, where $N$ is the normal force. Its value is not given by the friction law but by the dynamics

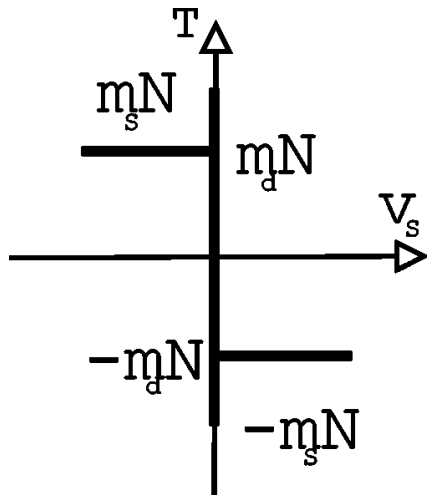

FIG. 2. Friction law, relating the friction force $T$ at a contact to the sliding velocity $v_{s}$, used in the simulations.

of the contacting particles. At the onset of sliding, the friction force drops discontinuously from $T_{s}= \pm \mu_{s} N$ to $T_{d}$ $= \pm \mu_{d} N$. It is easy to see that a single block drawn by a spring at constant velocity on a plane governed by this friction law undergoes a stick-slip motion such that the friction force $T_{e}$, every time the block comes to rest, is given by $T_{e}$ $=2 T_{d}-T_{s}$ and the block moves as an overdamped harmonic oscillator during a slip $[8,15]$.

We implemented the above friction law in the framework of a contact dynamics algorithm [20-22]. For our purpose in the present study, where both efficiency (long time simulations with various parameters) and accuracy (because friction is a major ingredient of granular materials) are equally important, the contact dynamics method provides a suitable approach since the contact forces are determined with no resort to repulsive elastic potentials or artificial viscous damping. For this reason, the time step used in contact dynamics simulations can be much larger compared to molecular dynamics simulations. Let us also remark that in all real experiments mentioned above the stiffness of the particles is by orders of magnitude larger than that of the driving mechanism so that the assumption of infinitely rigid particles is a good physical approximation in this respect.

We used different values for system parameters, as will be specified below. Most of the results presented below correspond to the following default values: $\mu_{s}($ wall-disk $)=0.5$, $\mu_{d}($ wall-disk $)=0.45, \quad \mu_{s}($ disk-disk $)=0.18, \quad \mu_{d}($ disk-disk $)$ $=0.1, k=5 \times 10^{3} \mathrm{~N} / \mathrm{m}, v=1 \mu \mathrm{m} / \mathrm{s}$. The coefficient of friction between the particles and the piston is zero. We simulated systems with up to 400 particles, but most of simulations were performed with a collection of 200 particles with an aspect ratio $\alpha \equiv H / L=1.3$ so that $L \simeq 12 D$, where $D$ is the mean particle diameter. Hence our system represents rather a narrow pipe. The computation time with a significantly larger system for a long time steady state is prohibitive.

\section{FLOW REGIMES}

\section{A. Intermittent flow}

When the system is in static equilibrium, the spring force $F_{k}$ along the vertical direction is balanced by the sum of the total weight $w$ of the particles, the weight of the piston $w_{p}$, 
and the friction forces $T_{L}$ and $T_{R}$ exerted by the left and right walls, respectively:

$$
F_{k}=w+w_{p}+T_{L}+T_{R}
$$

By "driving force" we refer to the force $F$ defined by

$$
F=F_{k}-w_{p}
$$

The latter is simply the total force at the bottom of the granular column. From Eq. (1), we see that this force actually represents the total friction force exerted by the walls on the granular column since $F-w=T_{L}+T_{R}$. Due to upward motion, the friction forces acting on the granular column are on average mobilized downward, so that $T_{L}+T_{R}>0$, and thus $F / w>1$. The evolution of $F$ with time reveals the nature of the flow. If the system remains at rest, $F$ increases linearly with time due to the steady motion of the translational stage which shortens the spring. If the system moves faster than the translational stage, the spring is unloaded and $F$ decreases.

One of our basic observations is that a slight difference between static and dynamic coefficients of friction is necessary in order to obtain an intermittent flow. Figure 3(a) shows one example of the evolution of $F$ as a function of the total displacement $y$ of the translational in units of the average particle diameter $D$ in a case where $\mu_{s}=\mu_{d}$ both for particle-particle and particle-wall contacts. The fluctuations correspond to a clear scale separation between a long loading phase followed by a short unloading phase, a feature that characterizes an intermittent flow. But a steady state is reached where the system moves as a block (with no particle rearrangements) and the driving force remains constant.

Figure 3(b) shows the evolution of $F$ in a case where a slight difference between static and dynamic coefficients of friction is introduced at the wall-particle contacts and the particle-particle contacts. We observe a stick-slip motion with a large variability of slip amplitudes $S \equiv F_{s}-F_{e}$ defined as the difference between the force before $\left(F_{s}\right)$ and after $\left(F_{e}\right)$ a slip event. We observe no purely "sticking" phase, the force building up nonlinearly with time. This creep motion is followed by slip events of various sizes. In the following we focus on this last case where irregular intermittent flow occurs.

\section{B. Transition to constant flow}

Since the particle interactions involve no (time, length, and force) scales, the characteristic scales of the system are imposed by external forces and the pushing mechanism. Let $g$ and $m$ be the gravity (pointing downward) and the total mass of the system (including both the particles and the piston), respectively. We have two time scales:

$$
t_{k}=\left(\frac{m}{k}\right)^{1 / 2}
$$

and
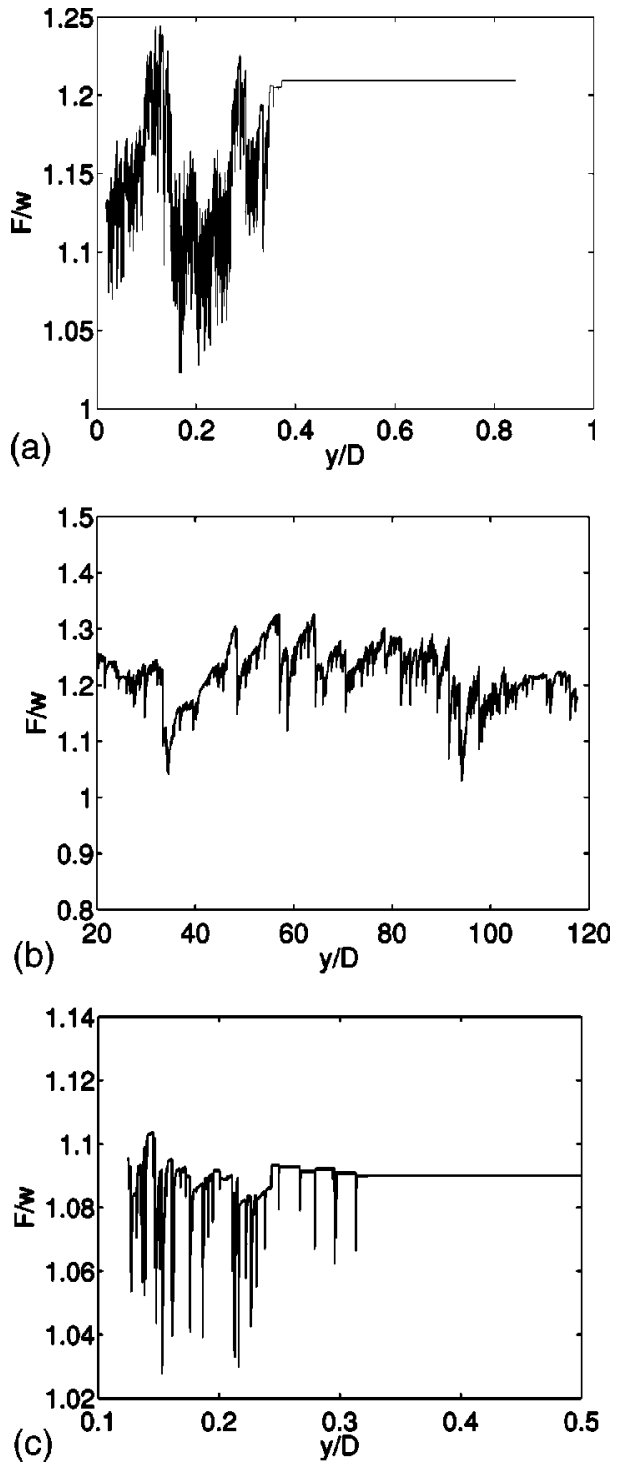

FIG. 3. Evolution of the driving force $F$, normalized by the total weight $w$ of the particles, as a function of cumulative displacement $y$, normalized by the mean particle diameter $D$; (a) $\mu_{s}=\mu_{d}=0.18$ for particle-particle contacts and $\mu_{s}=\mu_{d}=0.45$ for particle-wall contacts; (b) $\mu_{s}=0.18$ and $\mu_{d}=0.10$ for particle-particle contacts, $\mu_{s}$ $=0.50$ and $\mu_{d}=0.45$ for particle-wall contacts; (c) The case where the driving velocity $v$ is above the characteristic velocity.

$$
t_{g}=\frac{v}{g} .
$$

The time $t_{k}$ corresponds to the unloading time, i.e., the duration of a slip event. The time $t_{g}$ is a relaxation time, i.e., the time necessary for a particle initially at rest to reach the velocity $v$ under its own weight. Clearly, if $t_{g} \ll t_{k}$, i.e., when the pushing velocity is sufficiently low, the particle rearrangements during unloading will not be influenced by the pushing velocity. In the opposite case, the particle velocities are mainly dictated by the pushing velocity. The transition between these two regimes occurs for $t_{k}=t_{g}$, i.e., for a characteristic velocity $v_{c}$ given by 


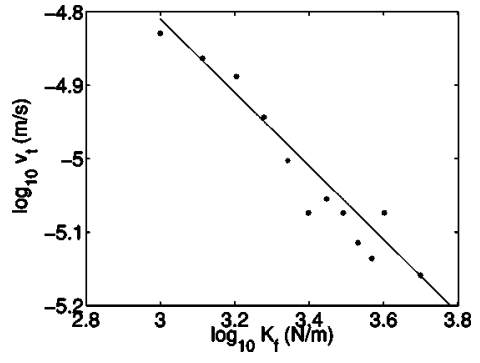

FIG. 4. The velocity $v_{t}$ at transition from intermittent to constant flow as a function of the stiffness $K_{f}$ of the pushing mechanism.

$$
v_{c}=\left(\frac{m}{k}\right)^{1 / 2} g .
$$

We find that a transition to constant flow indeed occurs when the driving velocity $v$ is larger than a transition velocity $v_{t} \simeq v_{c}$. In Fig. 3(c) is displayed the time series of the driving force $F$ when the driving velocity $v$ is increased beyond $v_{c}$ defined by Eq. (5). Instead of an intermittent flow [Fig. 3(b)] a constant flow is observed.

Figure 4 shows that $v_{t}$ decreases as $k^{-1 / 2}$ in agreement with Eq. (5). In the theoretical limit of $k=\infty, v_{t}$ is zero. There is no intermittent flow in this limit (unless a finite stiffness of the particles allows the system to store elastic energy). The transition to constant flow is discontinuous, i.e., the amplitude of slip events does not decrease continuously as $v$ approaches $v_{c}$. This is different from the behavior observed in Ref. [7] for a powder sheared in an annular cell, but is similar to the behavior observed in Ref. [15] for avalanches in a long rotating drum. This discontinuity might be related to the discontinuity of the friction force upon sliding at a contact, i.e., $\mu_{d}\left(v_{s}=0\right) \neq \mu_{s}$. In other words, as long as intermittent flow can take place, the force drop $S=F_{s}-F_{e}$ is basically controlled by the difference $\mu_{s}-\mu_{d}$ and not by the difference $v-v_{c}$.

It is worth emphasizing here that the nature of transition from intermittent to continuous flow in our simulations is fundamentally different from that in solid body friction. In the latter case, the transition is governed by the existence of a characteristic length scale $d_{0}$ so that the ratio $d_{0} / v$ defines an inertial time [14]. In our setup, there is no length scale inherent to contact interactions (the particle size being irrelevant in the limit considered here since $D / v \gg t_{k}$ ). The characteristic velocity $v_{c}$ is entirely dependent on external factors $k, g$, and $m$.

\section{EVOLUTION OF THE DRIVING FORCE}

\section{A. Mean force}

As can be seen in Fig. 3(b), the driving force $F$ in our simulations fluctuates in the range $[1.1 w, 1.3 w]$. The mean static driving force $F_{m}=\left\langle F_{s}\right\rangle$, where the average is taken over all forces $F_{s}$ just before slip events in the steady state, is $F_{m} \simeq 1.2 w$. From Eqs. (1) and (2), this implies that $\langle T\rangle$ $\equiv\left\langle T_{L}+T_{R}\right\rangle \simeq 0.2 w$.

The equilibrium of a packing in a vertical pipe can be analyzed by means of the Janssen model $[5,23]$. Each granu-

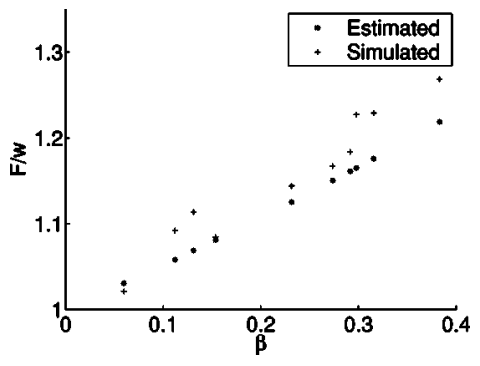

FIG. 5. The simulated driving force normalized by the weight of the particles, $F / W$, and the estimated value $\left(\exp ^{\beta}-1\right) / \beta$ as a function of $\beta$.

lar layer confined between two parallel horizontal sections of the material and the walls is equilibrated by the pressure drop across the layer (assumed to be uniform throughout the section) due to gravity and the friction forces exerted by the walls. This yields the total force $F_{m}$ at the bottom of a granular column (overcome by the driving force at incipient slip):

$$
F_{m}=\frac{e^{\beta}-1}{\beta} w,
$$

with

$$
\beta=2 K \mu_{w} \epsilon \alpha,
$$

where $\mu_{w}$ is the global (static) coefficient of friction of the material with the walls, $\alpha=H / L$ is the aspect ratio, and $K$ $=\sigma_{H} / \sigma_{L}$ is the normal stress ratio defined as the ratio of the normal horizontal stress $\sigma_{H}$ and the normal vertical stress $\sigma_{L}$. The indicator function $\epsilon$ is equal to 1 when the material is pushed upward (wall friction pointing downward) and -1 in downward failure (wall friction pointing upward). Equation (6) holds for $\beta \neq 0$, but, physically speaking, we have $F_{m}$ $=w$ in the case $\beta=0$ (no wall friction) which corresponds to the limit of the expression (6) of $F_{m}$ when $\beta \rightarrow 0$.

In order to evaluate the (static) coefficient of friction $\mu_{w}$ of the material with the walls, we take the average of the ratio of the friction force to the normal force at individual contacts with the walls. For most of contacts with the walls, the friction force is not fully mobilized. But, the average ratio of the friction force to the normal force at wall-particle contacts is on average equal to the ratio of the shear stress and the normal stress along the wall, and in this respect

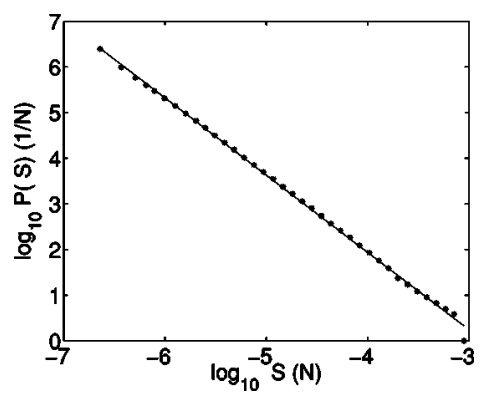

FIG. 6. Probability distribution $P$ of force drops $S$ during slip events. The solid line corresponds to a power law $P \propto S^{-b}$ with $b=1.7$. 

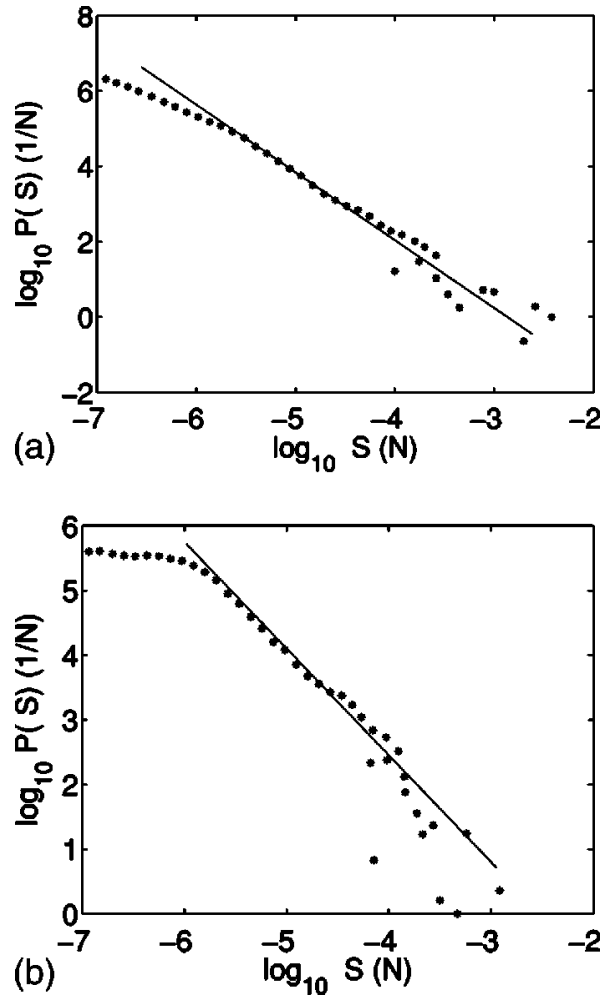

FIG. 7. Probability distribution $P$ of force drops $S$ during slip events for two sampling rates of the time series used in Fig. 6; (a) 10 times slower, (b) 50 times slower. The solid lines correspond to to a power law $P \propto S^{-b}$, where $b=1.7$.

represents correctly a macroscopic coefficient of friction. We find that $\mu_{w}$ varies between 0.030 and 0.095 in our simulations. This is much smaller than the particle-wall static coefficient of friction 0.5 and also smaller than the particleparticle static coefficient of friction 0.18 . The reason for such low values is rolling at the walls and the fact that not all the particles are moving in phase with the piston.

In order to estimate the value of the normal stress ratio $K$, we need the normal horizontal stress $\sigma_{H}$ at the bottom where we measure the normal vertical stress $\sigma_{L}=F_{m} / L$. Since, as we will see below, $\beta$ is small, the mean wall normal force varies on average almost linearly along the $y$ direction. Indeed, from Eq. (6), we have $F_{m} \simeq w \beta / 2$. As a result, $\sigma_{H}$ at the bottom is simply equal to $2 F_{H} / H$, where $F_{H}$ is the total normal force on a wall. We find $K \simeq 0.6$. This value of $K$ is almost equal to the limit of a passive Rankine state for which $K=(1-\sin \phi) /(1+\sin \phi)$, where $\phi \simeq 15^{\circ}$ is the internal angle of friction [23]. This means that, due to left-right symmetry of the setup, the major principal direction of the stress tensor is on average vertical (i.e., along the pushing direction), so that $\sigma_{L}$ and $\sigma_{H}$ correspond to the principal stresses.

For a set of simulations with all the parameters remaining the same but with different aspect ratios, the driving force $F$ normalized was plotted as a function of $\beta$ together with the estimated value; see Fig. 5. The parameter $\beta$ was calculated using an average value of $K$ and $\mu_{w}$ for each simulation. Although the estimated values seem to be slightly below the theoretical prediction of the Janssen model (this can be due to our evaluation of $K$ from the simulations), we see that they compare quite well on average although we deal here with a rather narrow pipe where the fluctuations and heterogeneities could be expected to mask the mean behavior.

\section{B. Distribution of force drops}

The probability distribution $P$ of force drops $S$ during slip events is shown in Fig. 6. We observe a nice power law $P(S) \propto S^{-b}$ with $b \simeq 1.7$ over nearly three decades. This distribution is extracted from more than $10^{6}$ slip events. The distribution shows no cutoff for very large slips. The sampling rate influences mainly the statistics in the range of small events.

Figure 7 shows the distribution $P(S)$ for the same time
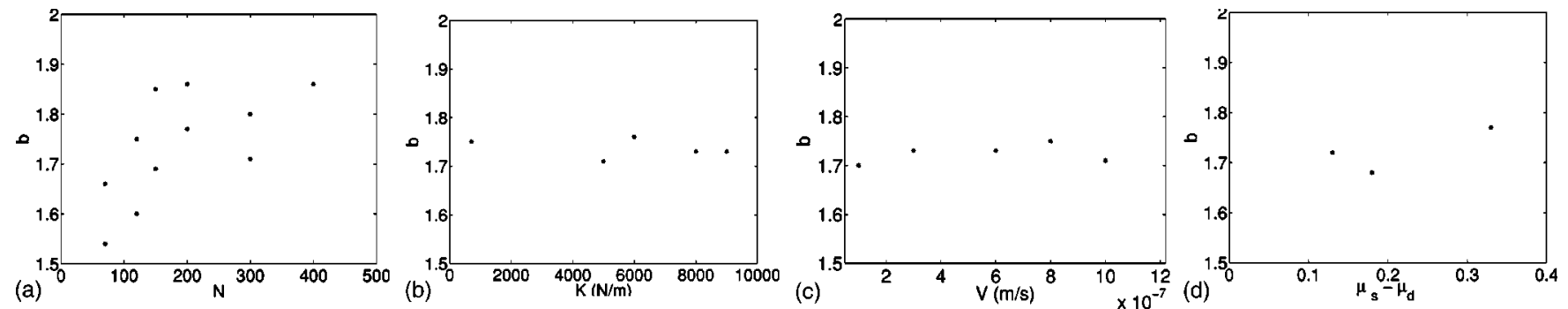

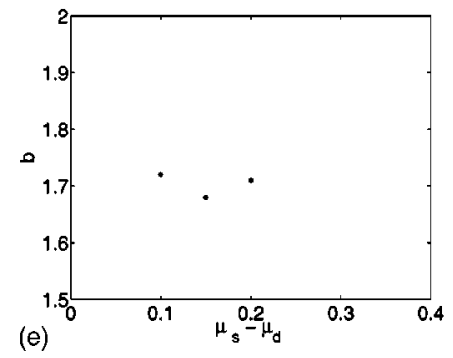

FIG. 8. The exponent $b$ of the power-law distribution of event sizes as a function of the number of particles $N$ (a), the spring stiffness $k$ (b), the driving velocity $v(\mathrm{c})$, and the difference $\mu_{s}-\mu_{d}$ between static and dynamic coefficients of friction for the disk-wall friction (d), and the difference $\mu_{s}-\mu_{d}$ between static and dynamic coefficients of friction for the disk-disk friction (e). 


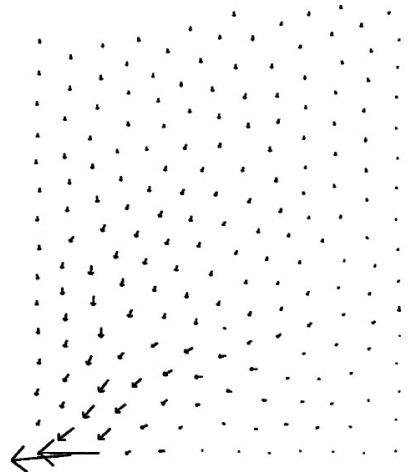

(a)

FIG. 9. (a) Displacements of particle centers with respect to the pushing piston during a large slip; (b) Variation of contact forces during the slip for reloaded contacts (black) and unloaded contacts (gray). The stippled lines are broken contacts.

series re-sampled at two different rates. For lower sampling rates, we get both fewer number of large events (so a broader scatter of the data) and fewer number of small events, but the value of $b$ (in the range where the power law is well defined) remains sensibly close to 1.7 . We further studied the influence of various system parameters on the distribution of slip sizes. In all cases we found a power law with an exponent always close to 1.7. In Figs. 8(a)-8(d) the exponent $b$ is plotted as a function of the number of particles $N_{p}$ (a), the spring stiffness $k$ (b), the driving velocity $v$ (c), and the difference $\mu_{s}-\mu_{d}$ between static and dynamic coefficients of friction (d). We note that each point corresponds to a separate simulation.

The power-law distribution of force drops with an exponent $b<2$ (as in earthquake events along faults) implies that the mean value of large slip events is not defined. The exponent is in the range suggested by the experiments of Kolb et al. [5] (with a lower statistical precision in those experiments). Let us also mention here the numerical simulations of Combe and Roux [24], where samples of two-dimensional frictionless particles were loaded biaxially. Under slowly increasing axial stress, they found a power-law distribution $\delta \varepsilon^{-1.46}$ for large axial strain increments corresponding to particle rearrangements between two successive equilibrium states of the sample. Like in those experiments, due to the absence of a mean, the force-displacement relationship in our simulations may be described as a Lévy process.

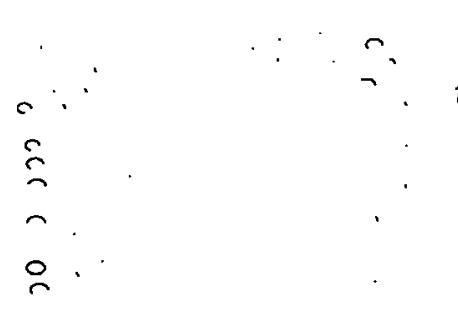

(a)

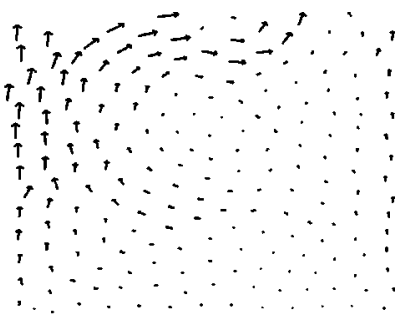

(b)

FIG. 10. Particle rotations (left) and the displacements of their centers (right) with respect to the pushing piston during a small slip event.

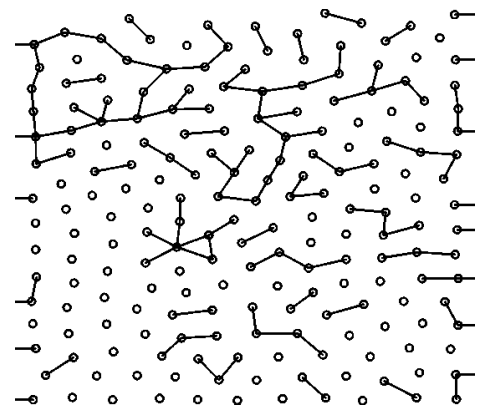

FIG. 11. A map of the positions of sliding contacts for a slip event.

\section{STRUCTURAL EVOLUTION}

The dynamic mobilization of friction at the walls and in the bulk reflects both the direction of the imposed motion of the piston and the correlated motions of the particles inside the cell. As a result, while the granular packing remains globally in a passive state $(F>w)$, the friction force evolves according to the dynamics of these collective rearrangements.

Figure 9(a) displays the displacements of particle centers with respect to the piston during a large slip. The particles move downward with respect to the piston and converge to the lower left corner of the sample. The particle displacements during a slip event can be as large as $0.05 \mathrm{D}$ corresponding to 12 times the displacement of the piston during the same event. The rearrangements are unstable and dissipate, in a very short time interval, the potential energy accumulated prior to the slip event. The energy dissipated due to inelastic collisions is often of the same order of magnitude as the energy dissipated at sliding contacts by friction forces! In Fig. 9(b) is shown the corresponding variation of the force network by distinguishing reloaded (force increase) and unloaded (force decrease) contacts. The stippled lines are broken contacts. We see that the slip event involves indeed a reorganization of contact forces, concentrated in this example mostly at the lower left corner, and the contact network itself. The forces vary here in the range from 0.01 to 1.4 times the force drop $S$.

As a result of the higher value of the coefficient of static friction at the walls with respect to the particle-particle contacts, most of particles roll on the walls. At those wall contacts the friction force is only partially mobilized. Figures 10(a) and 10(b) display the particle rotations and the displacements of their centers during a small slip event (with
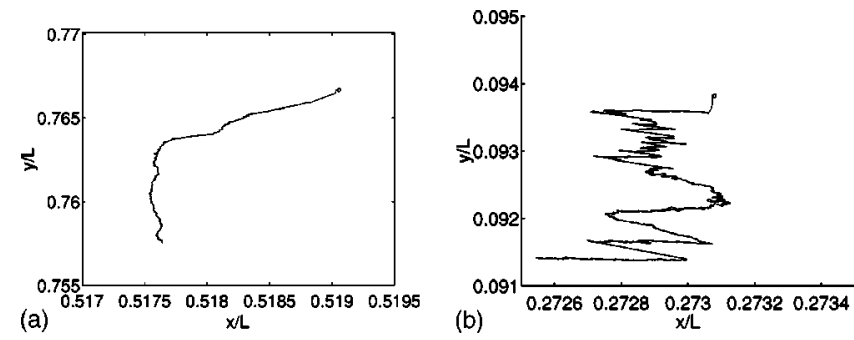

FIG. 12. The trajectories of two selected particles in the cell. 

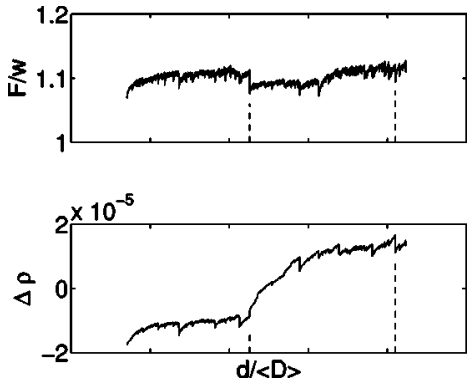

FIG. 13. A portion of a times series for the driving force $F$ (up) and the relative deviations $\Delta \rho / \rho$ of the solid fraction (down).

respect to the piston). We see that the largest rotations indeed take place at the walls and a convective motion of the particles occurs in the bulk. This corresponds to what sometimes is called a "rough wall" condition, implying that the strains occur basically in the bulk of the material [23]. In the same way, sliding contacts occur mainly in the bulk as shown in Fig. 11 where the positions of sliding contacts have been shown.

The convection patterns change continuously, but their average effect is to induce a diffusive motion of the particles as shown in Fig. 12 for two single particles in the medium. The cumulative effect of slip events gives rise to large diffusive displacements of the particles remixing the particles along the granular column. The erratic motions of the particles for much larger systems with homogeneous boundary conditions have been shown to be superdiffusive [25].

The variations of the solid fraction during slip events are of the order of $\Delta \rho / \rho \simeq 10^{-5}$. They can be positive (compaction) or negative (dilation). Since the solid fraction remains on average constant in the steady state (around 0.83), several successive dilatant events are usually followed by a contractant event. Figure 13 shows a portion of a times series for $F$ and $\Delta \rho / \rho$. The behavior of the solid fraction is clearly correlated with the force variations. Among hundreds of slip events occurring during the time interval shown in this figure, we can discern several rather large events where the force drop is more pronounced. Considering only these large events, we see that most of slip events (force drops) correspond to a decrease of the solid fraction. However, it happens occasionally that the solid fraction increases during a slip event. We have marked on the figure two such large slip events where the solid fraction increases for the first one and decreases for the second.

\section{CONCLUSION}

In this paper, we studied the slow steady-state flow of a granular column inside a narrow pipe. The finite stiffness of the pushing mechanism entails a strongly irregular intermittent flow when the contacts are governed by a velocity weakening friction law. We found the following: (i) A transition to constant flow occurs for a driving velocity $v_{t}=(\mathrm{m} / \mathrm{k})^{1 / 2} \mathrm{~g}$ involving no internal length and times scales; (ii) The mean static driving force is correctly predicted by the Janssen model in spite of the small size of our systems; (iii) The distribution of force drops during slip events falls off as a power law over three decades with an exponent which does not seem to depend strongly on system parameters; (iv) The slip events are preceded by creep motion leading most of time to a small increase of the solid fraction, whereas slip events generally involve a dilation of the material.

The origin of the broad distribution of event sizes remains an open question. Clearly, the upward motion of the translational stage tends to destabilize gravity-induced arches in the system, whereas friction mobilization at the walls resists motion. This competition between the mobilization of friction at the walls and gravity-induced arching, which act in opposite directions, is likely to be at the origin of large slip events. The system size is such that the walls can considerably enhance the fluctuations. Although the focus of this paper was basically on a narrow pipe, it is interesting to evaluate the influence of the walls for larger systems. However, from a strictly numerical point of view, such an extension of this work requires many more particles and more involved computations for a correctly represented steady state.
[1] A. Ngadi and J. Rajchenbach, Phys. Rev. Lett. 80, 273 (1998).

[2] I. Albert, P. Tegzes, B. Kahng, R. Albert, J. G. Sample, M. Pfiefer, A.-L. Barabási, T. Vicsek, and P. Schiffer, Phys. Rev. Lett. 84, 5122 (2000); I. Albert, J. G. Sample, A. J. Mross, S. Rajagopalan, A.-L. Barabási, and P. Schiffer, Phys. Rev. E 64, 061303 (2001).

[3] S. Nasuno, A. Kudrolli, and J. P. Gollub, Phys. Rev. Lett. 79, 949 (1997).

[4] J.-C. Géminard, W. Losert, and J. P. Gollub, Phys. Rev. E 59, 5881 (1999).

[5] E. Kolb, T. Mazozi, E. Clément, and J. Duran, Eur. Phys. J. B 8, 483 (1999).

[6] G. Ovarlez, E. Kolb, and E. Clément, Phys. Rev. E 64, 060302 (2001).

[7] M. Lubert and A. de Ryck, Phys. Rev. E 63, 021502 (2001).
[8] F. Radjai, P. Evesque, D. Bideau, and S. Roux, Phys. Rev. E 52, 5555 (1995).

[9] B. Dulieu and F. Radjai, in Friction, Arching, Contact Dynamics, edited by D. Wolf and P. Grassberger (World Scientific, Singapore, 1997), p. 281.

[10] R. A. Bagnold, Proc. R. Soc. London, Ser. A 295, 219 (1966).

[11] P. Evesque, Phys. Rev. A 43, 2720 (1991).

[12] F. P. Bowden and D. Tabor, The Friction and Lubrification of Solids, Part I (Clarenden Press, Oxford, 1950); The Friction and Lubrifrication of Solids, Part II (Clarenden Press, Oxford, 1964).

[13] B. N. J. Persson, Sliding Friction, Physical Principles and Applications (Springer-Verlag, Berlin, 1998).

[14] F. Heslot, T. Baumberger, B. Perrin, and C. Caroli, Phys. Rev. E 49, 4973 (1994). 
[15] M. Caponeri, S. Douady, S. Fauve, and C. Laroche, in Mobile Particulate Systems, edited by E. Guazzelli and L. Oger (Kluwer, Dordrecht, 1995) pp. 331-366.

[16] J. M. Carlson, J. S. Langer, and B. E. Shaw, Rev. Mod. Phys. 66, 657 (1994).

[17] P. Bak, C. Tang, and K. Wiesenfeld, Phys. Rev. Lett. 59, 381 (1987).

[18] V. Frette, K. Christensen, A. Malthe-Sorenssen, J. Feder, T. Jossang, and P. Meakin, Nature (London) 379, 49 (1996).

[19] K. Christensen, A. Corral, V. Frette, J. Feder, and T. Jossang, Phys. Rev. Lett. 77, 107 (1996).
[20] J. J. Moreau, Eur. J. Mech. A/Solids 13 (no. 4 suppl.), 93 (1994).

[21] M. Jean, in Mechanics of Geometrical Interfaces, edited by A. P. S. Selvaduri and M. J. Boulon (Elsevier, Amsterdam, 1995), p. 463.

[22] F. Radjai, Comput. Phys. Commun. 121-122, 294 (1999).

[23] R. M. Nedderman, Statics and Kinematics of Granular Media (Cambridge University Press, Cambridge, England, 1992).

[24] G. Combe and J. N. Roux, Phys. Rev. Lett. 85, 3628 (2000).

[25] F. Radjai and S. Roux (2002), Phys. Rev. Lett. 89, 064302 (2003). 